\title{
Helminths of the sand lizard, Lacerta agilis (Reptilia, Lacertidae), in the Palaearctic: faunal diversity and spatial patterns of variation in the composition and structure of component communities
}

\author{
V. P. SHARPILO ${ }^{1}$, V. BISERKOV ${ }^{2}$, A. KOSTADINOVA ${ }^{2}$, J. M. BEHNKE K $^{3 *}$ \\ and $\mathrm{Y}$. I. KUZM IN ${ }^{1}$ \\ ${ }^{1}$ Department of Parasitology, I.I. Schmalhausen Institute of Zoology, National Academy of Sciences of Ukraine, \\ 15 Bogdan Khmelnitsky Street, Kiev-30 MSP 01601, Ukraine \\ ${ }^{2}$ Department of Biodiversity, Central Laboratory of General Ecology, Bulgarian Academy of Sciences, 2 Gagarin Street, \\ 1113 Sofia, Bulgaria \\ ${ }^{3}$ School of Life and Environmental Sciences, University of Nottingham, University Park, Nottingham NG7 2 RD
}

(Received 14 March 2001; accepted 8 April 2001)

SUMMARY

We studied variation in the structure of component communities of helminths in sand lizards, Lacerta agilis, from 30 localities in the Ukraine and Bulgaria. Thirty-five separate samples of lizards, with a total of 661 completely censused infracommunities, yielded 30 helminth species ( 4 cestodes, 10 trematodes, 3 acanthocephalans and 13 nematodes). In its range within the Ukraine, L. agilis serves as the final host for 13 species of which only 3 (S. lacertae, S. hoffmanni and $P$. molini) can be considered as lizard specialists. A characteristic feature of these helminth component communities was the large proportion of heteroxeneous helminth species for which L. agilis serves as paratenic host. Sand lizards in the meadow steppeland zone were primarily parasitized by larval helminths that represented a major proportion of the total number of all worms recovered while those sampled in the grassland/forest transition zone were characterized by substantially higher proportions of adult helminths using lizards as final hosts. However, L. agilis was parasitized by a much higher proportion of lizard specialists in the 'typical' habitats of the meadow steppeland zone as opposed to those located in 'marginal' habitats in the grassland/forest transition zone, where helminths were shared to a greater extent with amphibian hosts.

Key words: sand lizard, Lacerta agilis, helminth fauna, nematodes, cestodes, trematodes, helminth communities, regional variation, Ukraine, Bulgaria.

\section{INTRODUCTION}

The community ecology of parasites of vertebrates is a subject that has attracted considerable interest in the last decade, resulting in the development of a conceptual framework for the hierarchical structure of parasite communities (Poulin, 1998a). This has enabled hypotheses to be formulated about the key processes that regulate the composition and structure of parasite component communities (Esch, Bush \& Aho, 1990). Although fish, birds and mammals have been widely studied in this context, relatively little information is available on the characteristics of helminth communities in reptiles, especially with regard to quantitative data based on completely censused infracommunities (Aho, 1990; Bush, 1990).

* Corresponding author: School of Life and Environmental Sciences, University of Nottingham, University Park, Nottingham NG7 2RD. Tel: +0115951 3208. Fax: +0115 951 3251. E-mail: jerzy.behnke@ nottingham.ac.uk
Moreover, the structure of helminth communities in reptiles from the Palaearctic region has received little attention at both hierarchical levels of organization, the infracommunity and the component community.

One of the most abundant and widespread reptile species in Eurasia is the sand lizard, L. agilis, its distribution being primarily attributable to its tolerance of diverse environments ranging from the steppe through agricultural fields to woodland habitats (Yablokov, Baranov \& Rozanov, 1981; Frazer, 1989). Nevertheless, as with most reptile species, the movement of individuals is thought to be confined to a considerably more limited range since, being territorial animals, sand lizard individuals or pairs occupy, mark and defend a limited area within the habitat (Lewin, 1992). The helminth fauna of $L$. agilis has been described throughout its range in Europe, and is reported to be the richest among the Palaearctic lacertids (Sharpilo, 1976). The widespread distribution of sand lizards, and the available wealth of information on their biology, ecology and parasite fauna, make this species a particularly useful 


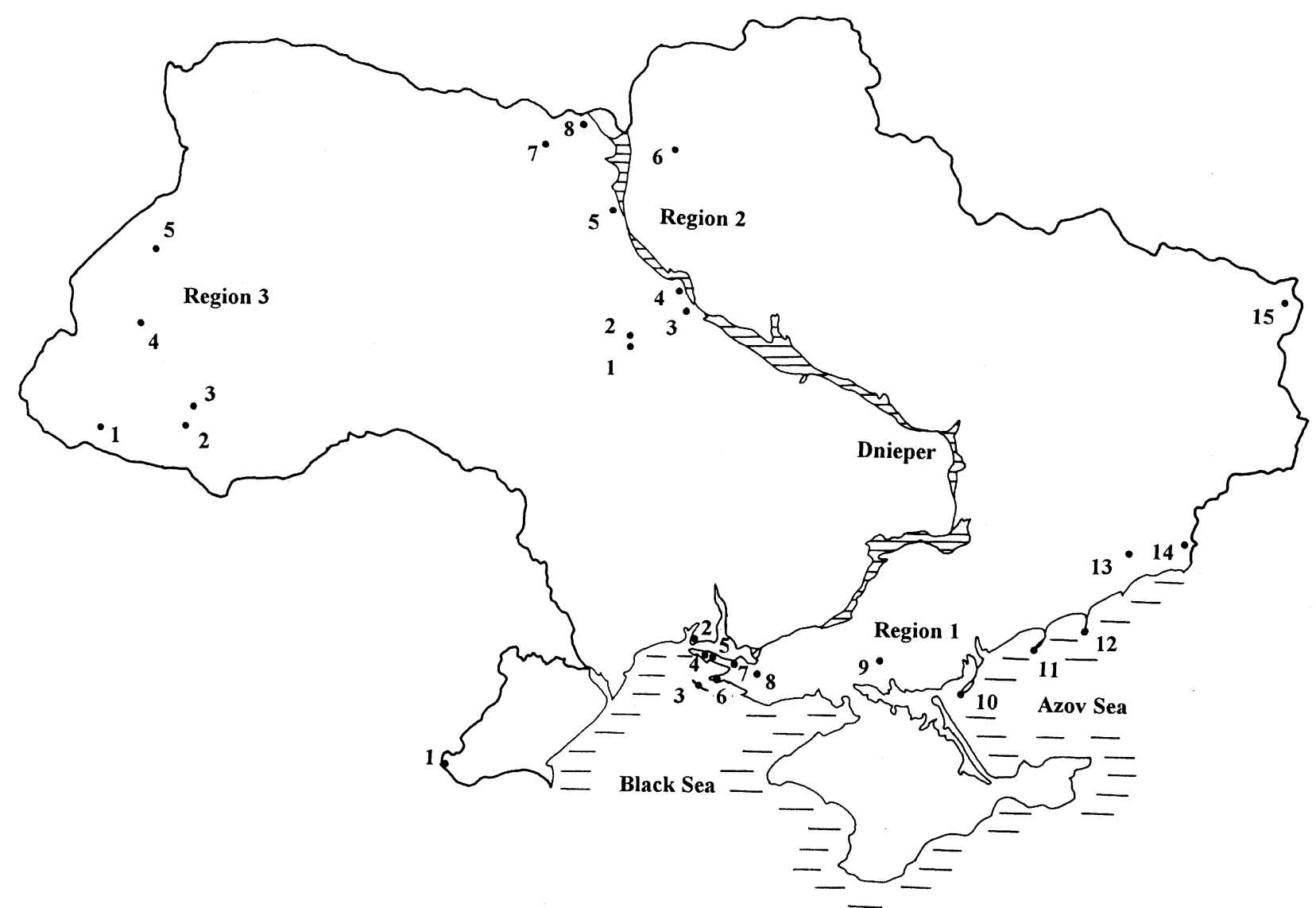

Fig. 1. Map of the Ukraine showing the localities in the 3 regions where Lacerta agilis was sampled. The numbers within each region (R1, R2 and R3) correspond to individual sampling sites, as listed in the Appendix.

and relevant host for detailed studies of the community ecology of their parasites. Sand lizards occur in habitats of contrasting quality and over a wide geographical range, providing a further avenue for the elucidation of the processes that regulate helminth community structure in relation to habitat type and at both local and regional levels.

In this paper, we report on the helminth fauna of the sand lizard, L. agilis, from numerous localities in the Ukraine and Bulgaria, exploiting the largest data set (based on fully censused infracommunities) collected for any reptile species within the Palaearctic to test the specific hypotheses that the parasite communities of lizards from 'typical' habitats should be dominated by lizard specialists and should be highly predictable, whilst those from lizards living in 'marginal habitats' should be dominated by host generalist species and should be less predictable. Our approach is to analyse the regional patterns of variation in helminth component communities in sand lizards from different zones within the region, encompassing the meadow steppeland areas of the Ukraine, where these lizards are considered to have originated, and marginal zones represented by the grassland/forest transition phytogeographical areas and the Carpathian mountains in the Ukraine. For each zone we analyse the structure and similarity of composition of helminth communities at both infraand component community levels.

\section{MATERIALS AND METHODS}

In total 32 batches of lizards were collected in a large-scale field study by one of the authors (V. P. Sharpilo) during the warm months of the years between 1955 and 1991 from 28 localities in the Ukraine. Three batches were collected in 1984-1985 from 2 localities in southwestern Bulgaria. As a result, a data set containing the individual hostparasite records of a total of $661 \mathrm{~L}$. agilis was available for analysis. The locations of all of the study sites within the 3 regions in the Ukraine are shown in Fig. 1. These will be referred to as follows: (i) Region 1 (R1), meadow steppeland region (15 localities, 18 samples [site N10 was sampled 3 times and site N13 twice, see Appendix], 342 specimens, from the Black and Azov Sea coasts and short grass steppes in the east of the Ukraine); (ii) Region 2 (R2), middle Dnieper reaches along Kiev's and Kanev's dams (8 localities, 9 samples [site N1 was sampled twice, see Appendix] 172 specimens) and (iii) Region 3 (R3), Carpathian Mountains region (5 localities, 5 samples, 100 specimens) (see Appendix for additional sample data). The latter 2 regions 
correspond to the grassland/forest transition phytogeographical zone. Bulgarian samples were collected in 2 localities in southwest Bulgaria (Pirin mountains), Yavorov hut (39 specimens, sampled twice) and village Razlog (3 specimens). Therefore only 3 localities in the Ukraine were sampled more than once and 1 in Bulgaria.

Although collected over a long period, the data set represents a series of homogeneous samples (see Janovy, Clopton \& Percival, 1992) since each sample includes hosts collected at 1 locality within 1 day and none of the community parameters showed significant association with the month of collection. Hosts were examined for parasites using standard techniques and each helminth infracommunity was fully censused. All helminths were identified and counted. Voucher material is deposited in the collection of the I.I. Schmalhausen Institute of Zoology, National Academy of Sciences, Kiev, Ukraine.

Ecological terms are used according to those of Bush et al. (1997). Prevalences of $10 \%$ for sample size $>15$ and of $20 \%$ for sample size $<15$ hosts were adopted as the lowest limits in identifying the component species within each sample (see Bush, Aho \& Kennedy, 1990). Species with prevalences of $50 \%$ or higher were assigned as core species (see Aho, 1990) in all samples. The infracommunity data were used to calculate the mean number of helminth species and worms, the mean Brillouin's diversity index (HB, values calculated using natural logarithms), and the mean Berger-Parker dominance index (BP) (Magurran, 1988).

Jaccard's binary coefficient for qualitative (presence/absence) data and percentage similarity index for quantitative data (Krebs, 1989) were used as similarity measures. The similarities were calculated using all possible comparisons at both the infracommunity and component community levels. All similarity and diversity measures were calculated using the program BIODIV (Baev \& Penev, 1995). For statistical comparisons standard non-parametric tests (Spearman rank correlation $\left(r_{s}\right)$, Kruskal-Wallis $(\mathrm{K}-\mathrm{W})$ and Mann-Whitney (M-W) tests) were applied (Sokal \& Rohlf, 1981).

Sample size from the various localities in the Ukraine varied, typically comprising more than 15 lizards although some samples were lower (especially in R1) but only 4 samples comprised fewer than 10 lizards. Since sample size only significantly correlated with the total number of species $\left(r_{s}=0 \cdot 384\right.$, $n=32, \quad P<0.05)$, we used the residuals of a regression of species richness on sample size to estimate component community richness with sampling effort taken into account (see Poulin, $1998 b)$. Finally, in addition to the non-parametric tests, we adopted multiple regression (data $\log (x+1)$ transformed) for assessing functional relationships among community parameters while correcting for sample size.

\section{RESULTS}

\section{The helminth fauna of $\mathrm{L}$. agilis}

Thirty species of helminths were recorded among the total sample size of 661 lizards (4 cestodes, 10 trematodes, 3 acanthocephalans and 13 nematodes). A list of the species, arranged according to their lifecycles, plus the main final host groupings, are presented in Table 1.

For 13 of the helminths L. agilis served as the final host. Three can be considered as lizard specialists, the nematodes $S$. lacertae and $S$. hoffmanni, and the trematode $P$. molini (see Sharpilo, 1976). Two other species parasitize a larger range of reptiles, $O$. tuberculata (25 reptile species belonging to 12 genera) and Telorchis sp. (9 reptile species belonging to 7 genera, data from Sharpilo, 1976). Two species, $O$. filiformis and Nematotaenia sp., are common parasites of both amphibians and reptiles. The remaining trematode species are either parasites of amphibians or birds, and are only incidentally found in reptiles (see Table 1).

Nearly $60 \%$ of the species, recovered in L. agilis represented larval forms. The sand lizard serves as paratenic host for the majority of these helminths (14 species, including 3 new host records, Table 1) which complete their life-cycles either in avian or mammalian final hosts, the only exception being $S$. contortus which matures in water turtles. Three species use $L$. agilis as an intermediate host. However, the sand lizard is a complementary host in this case since the main intermediate hosts of these helminths are amphibians and rodents (Sharpilo, 1983).

\section{Regional differences in the helminth fauna of \\ L. agilis}

Lizards from the 3 regions in the Ukraine differed in their helminth fauna when viewed at the class level. Although nematodes were represented similarly in the species regional lists $(50,46.7$ and $40 \%$ of the total number of species in R1, R2 and R3, respectively), trematode infections differed $(23 \cdot 1,46 \cdot 7$ and $40 \%$, respectively) with a particularly low prevalence in R1. However, more cestode species were recovered in $\mathrm{R} 1$ (15.4, 6.6 and $10 \%$ respectively) and no acanthocephalan species were found in R2 (vs. 11.5 and $10 \%$ in R1 and R3, respectively). Bulgarian samples contained only 1 cestode (Mesocestoides spp.) and 1 nematode (P. clausa).

At the species level, the helminth fauna of the 4 regions studied in the Ukraine and Bulgaria exhibited low similarity, the Jaccard qualitative similarity index ranging between 0.06 and 0.47 (Fig. 2). The species composition of the helminths found in Bulgaria was quite distinct from the 3 Ukrainian regions, and the 3 regions in the Ukraine grouped 
Table 1. Helminth fauna of Lacerta agilis from the Ukraine and Bulgaria

\begin{tabular}{|c|c|c|c|c|c|}
\hline Species & $\begin{array}{l}\text { NCOM/ } \\
\text { Region }\end{array}$ & NCORE & $\begin{array}{l}\text { Stage/Host } \\
\text { category }\end{array}$ & Life-cycle & Final hosts \\
\hline \multicolumn{6}{|l|}{ Trematoda } \\
\hline Plagiorchis molini Lent et Freitas, (1940) & $3 / \mathrm{R} 2, \mathrm{R} 3$ & 1 & $\mathrm{~A} / \mathrm{FS}$ & $\mathrm{C}$ & Lacertidae \\
\hline P. elegans (Rudolphi, 1802) & $3 / \mathrm{R} 1$ & 0 & $\mathrm{~A} / \mathrm{F}$ & $\mathrm{C}$ & Birds \\
\hline P. maculosus (Rudolphi, 1802) & 0 & 0 & $\mathrm{~A} / \mathrm{F}$ & $\mathrm{C}$ & Birds \\
\hline Pleurogenoides medians (Olsson, 1876) & $2 / \mathrm{R} 1$ & 0 & $\mathrm{~A} / \mathrm{F}$ & $\mathrm{C}$ & Amphibians \\
\hline Prosotocus confusus (Looss, 1894) & 0 & 0 & $\mathrm{~A} / \mathrm{F}$ & $\mathrm{C}$ & Amphibians \\
\hline Pleurogenes claviger (Olfers, 1816) & 0 & 0 & $\mathrm{~A} / \mathrm{F}$ & $\mathrm{C}$ & Amphibians \\
\hline Telorchis sp. & 0 & 0 & $\mathrm{~A} / \mathrm{F}$ & $\mathrm{C}$ & $\begin{array}{l}\text { Water snakes } \\
\& \text { turtles }\end{array}$ \\
\hline Brachylaima sp & 0 & 0 & $\mathrm{~J} / \mathrm{F}$ & $\mathrm{C}$ & Birds \\
\hline Strigea sp. (tetracotyle) & 0 & 0 & $\mathrm{~L} / \mathrm{I}$ & $\mathrm{C}$ & Birds \\
\hline Alaria alata (mesocercaria) & 0 & 0 & $\mathrm{~L} / \mathrm{P}$ & $\mathrm{C}$ & Mammals \\
\hline \multicolumn{6}{|l|}{ Cestoda } \\
\hline Oochoristica tuberculata (Rudolphi, 1819) & $6 / \mathrm{R} 1$ & 0 & $\mathrm{~A} / \mathrm{F}$ & $\mathrm{C}$ & Reptiles \\
\hline Nematotaenia sp. & $1 / \mathrm{R} 1$ & 0 & $\mathrm{~A} / \mathrm{F}$ & $\mathrm{C}$ & $\begin{array}{l}\text { Amphibians } \\
\text { and reptiles }\end{array}$ \\
\hline Mesocestoides spp. & 1/R1 & 0 & $\mathrm{~L} / \mathrm{I}$ & $\mathrm{C}$ & Mammals \\
\hline Cladotaenia circi Yamaguti (1935) & 0 & 0 & $\mathrm{~L} / \mathrm{I}$ & $\mathrm{C}$ & Birds \\
\hline \multicolumn{6}{|l|}{ Nematoda } \\
\hline Oswaldocruzia filiformis (Goeze, 1782) & 12/R1,R2,R3 & 7 & $\mathrm{~A} / \mathrm{F}$ & $\mathrm{D}$ & $\begin{array}{l}\text { Amphibians } \\
\text { and reptiles }\end{array}$ \\
\hline Spauligodon lacertae Sharpilo, (1966) & 1/R1 & 1 & $\mathrm{~A} / \mathrm{FS}$ & $\mathrm{D}$ & Lacertidae \\
\hline Skrjabinelazia hoffmanni Li (1934) & $2 / \mathrm{R} 1$ & 2 & $\mathrm{~A} / \mathrm{FS}$ & $\mathrm{D}$ & Lacertidae \\
\hline Physaloptera clausa (Rudolphi (1819) & $5 / \mathrm{R} 1$ & 1 & $\mathrm{~L} / \mathrm{P}$ & $\mathrm{C}$ & Mammals \\
\hline Spiroxys contortus (Rudolphi, 1819) & 0 & 0 & $\mathrm{~L} / \mathrm{P}$ & $\mathrm{C}$ & Turtles \\
\hline Habronema sp. & 1/R1 & 0 & $\mathrm{~L} / \mathrm{P}$ & $\mathrm{C}$ & Mammals \\
\hline Physocephalus sexalatus (Molin, 1860) & $1 / \mathrm{R} 3$ & 0 & $\mathrm{~L} / \mathrm{P}$ & $\mathrm{C}$ & Mammals \\
\hline Ascarops strongylina (Rudolphi, 1819) & $2 / \mathrm{R} 2$ & 0 & $\mathrm{~L} / \mathrm{P}$ & $\mathrm{C}$ & Mammals \\
\hline Spirocerca lupi (Rudolphi, 1819) & $1 / \mathrm{R} 2$ & 0 & $\mathrm{~L} / \mathrm{P}$ & $\mathrm{C}$ & Mammals \\
\hline Cosmocephalus obvelatus (Creplin, 1825) & 0 & 0 & $\mathrm{~L} / \mathrm{P}^{*}$ & $\mathrm{C}$ & Birds \\
\hline Paracuaria adunca (Creplin, 1846) & 1/R1 & 0 & $\mathrm{~L} / \mathrm{P} *$ & $\mathrm{C}$ & Birds \\
\hline Pteygodermatites cahirensis (Jagerskiold, 1904) & 2/R1 & 0 & $\mathrm{~L} / \mathrm{P} *$ & $\mathrm{C}$ & Mammals \\
\hline Acuariidae gen. sp. & $5 / \mathrm{R} 1$ & 0 & $\mathrm{~L} / \mathrm{P}$ & $\mathrm{C}$ & Birds \\
\hline \multicolumn{6}{|l|}{ Acanthocephala } \\
\hline Sphaerirostris picae (Rudolphi, 1819) & 10/R1 & 3 & $\mathrm{~L} / \mathrm{P}$ & $\mathrm{C}$ & Birds \\
\hline Centrochynchus sp. & $3 / \mathrm{R} 1$ & 1 & $\mathrm{~L} / \mathrm{P}$ & $\mathrm{C}$ & Birds \\
\hline Moniliformis moniliformis (Bremser, 1811) & 0 & 0 & $\mathrm{~L} / \mathrm{P}$ & $\mathrm{C}$ & Mammals \\
\hline
\end{tabular}

* New host record.

A, adult; J, juvenile; L, larval; C, Complex, D, direct; P, paratenic; I, intermediate; F, final; S, lizard specialist; $\mathrm{NCOM}=$ no. of communities where the species is component; NCORE $=$ no. of communities where the species is core.

according to their location in the meadow steppeland zone (R1) and the grassland/forest transition zones (R2 and R3).

A comparison of the structure of helminth fauna between the 3 regions in the Ukraine in relation to the functional role of $L$. agilis in the helminth lifecycles, revealed a similar grouping. Sand lizards in the meadow steppeland zone (R1) were primarily parasitized by larval helminths which represented $80.8 \%$ of the total number of all worms recovered from these samples; of these, helminths maturing in mammals dominated numerically $(63.6 \%$ of worms, respectively, Fig. 3A). On the other hand, the 2 regions in the grassland/forest transition zone (R2 and R3) were characterized by substantially higher proportions of helminths using lizards as final hosts $(65.1$ and $96.3 \%$ of worms, respectively).
When only helminths whose adult stages were found in L. agilis were analysed (Fig. 3B), the R1 exhibited a high proportion of species specializing in parasitizing lizards and other reptiles $(78.0 \%)$, while the latter 2 regions showed high proportions of the helminths using amphibians as the main hosts (81 and $84.2 \%$ of worms, respectively).

\section{Helminth communities in $\mathrm{L}$. agilis from the Ukraine}

The species composition of helminth communities, as well as prevalence of the species infecting $L$. agilis, varied across the samples from the Ukraine (Tables 2 and 3). Most species occurred at low prevalence so that only 19 out of the 30 species recorded in this study could be considered component species in at 


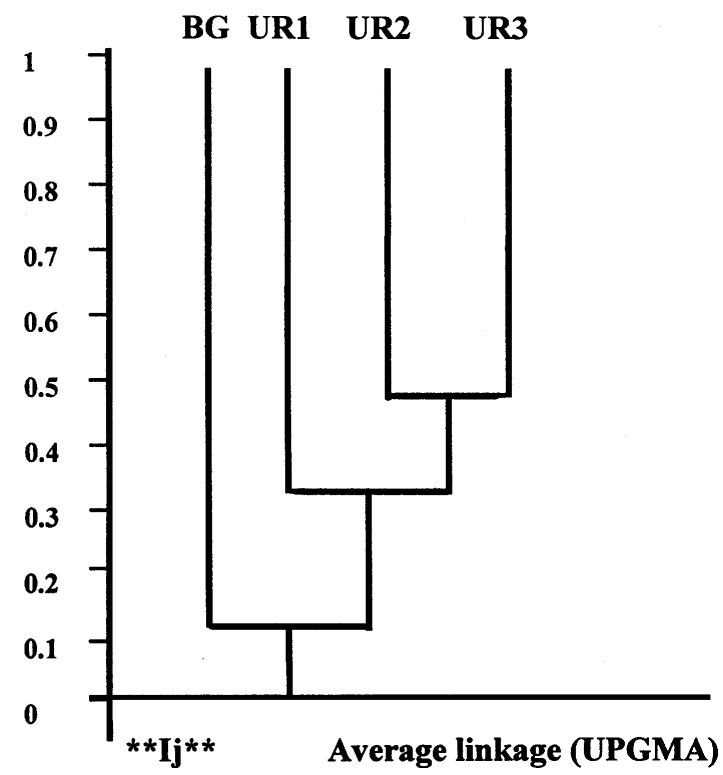

Fig. 2. Dendrograms (UPGMA) showing the qualitative similarity between helminth faunas of Lacerta agilis sampled in Bulgaria (BG) and the 3 regions in the Ukraine (UR1, UR2 and UR3).

least 1 sample and of these 7 species attained core status in at least 1 of these samples.

The list of the component/core species of the Meadow steppeland region was characteristic in that here substantially more species were recovered at appreciable prevalence (14 component/5 core) compared with those from the grassland/forest transition zone (R2 and R3, 5/1 and 3/2, respectively, Tables 1,2 and 3 ). The identity of the core species also differed. Within the first region (R1) 2 lizard specialists and 3 larval helminths reached core status (S. hoffmanni, S. lacertae, S. picae, Centrorhynchus sp. and $P$. clausa), while in the 2 regions representing grassland/forest transition zones only the amphibian/reptile generalist $O$. filiformis and the lizard specialist $P$. molini were considered core species.

Helminth communities in L. agilis from the Ukraine exhibited wide variations in species richness (Table 4). The total number of species ranged from 1 (2 samples) to 7 (4 samples), however, richness based on species consistently present in the community (i.e. component species) did not exceed 2 in $21(66 \%)$ samples although in 4 samples it was 4 (overall range $0-4$ ).

Despite 15 samples yielding core species, overall the infracommunity richness and abundance were very low. The mean number of species per host did not exceed 1 in $20(63 \%$ ) samples (range 0.13-1.64) and the mean number of individual worms per host did not exceed 10 in $27(84 \%)$ samples (range 0-14-645). Similarly, the values for diversity, although calculated for infected hosts only, were low (means equaling 0 in $8(25 \%)$ samples) but the dominance levels (Berger-Parker Index) were notably high (means exceeding 0.7 in all samples).
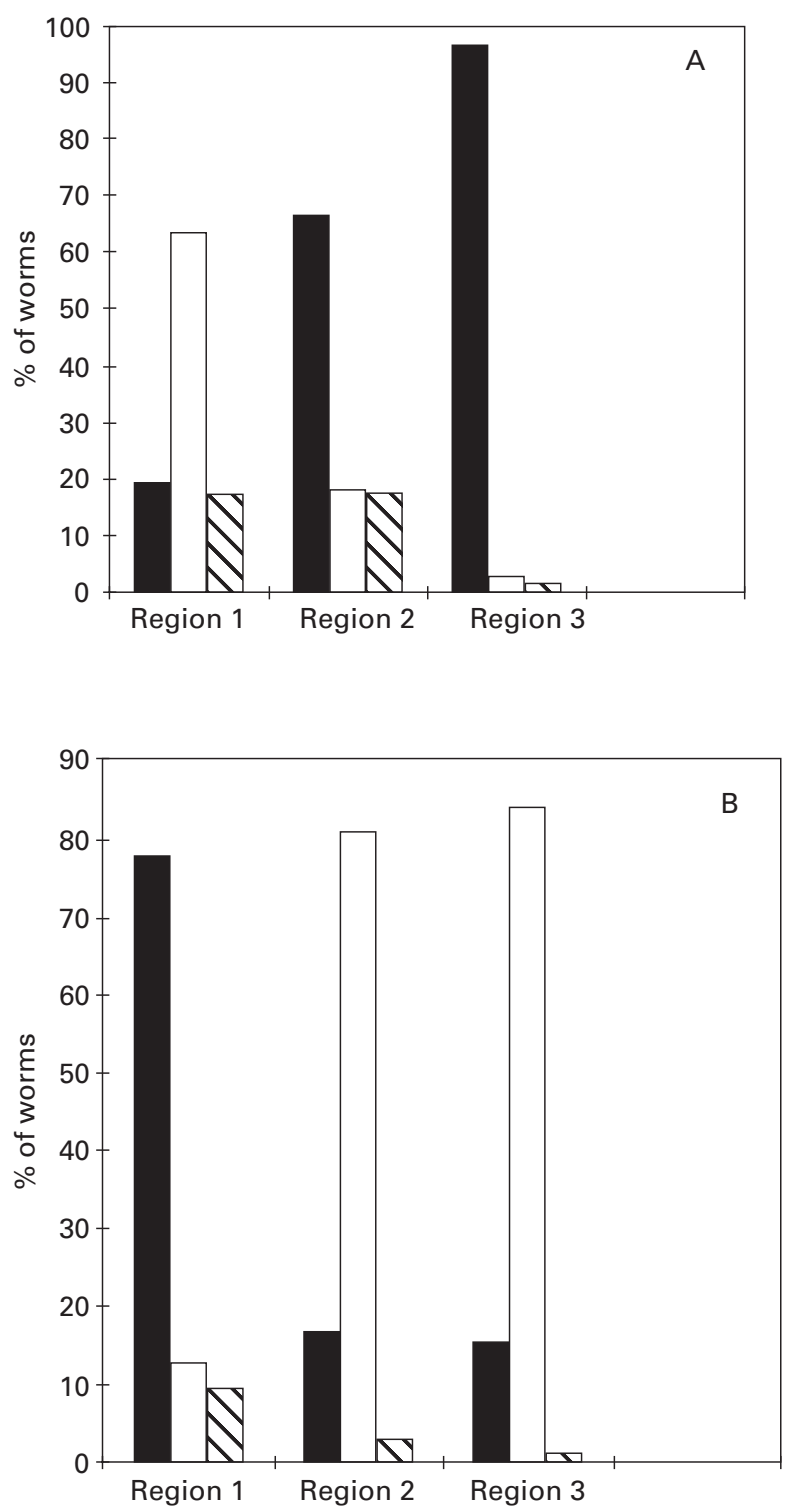

Fig. 3. Structure of the helminth fauna of Lacerta agilis in the 3 regions in the Ukraine. (A) Relative proportions (percent of total number of worms recovered) of adult and larval helminths ( $\square$, adult helminths; $\square$, larval helminths maturing in mammals; $\mathbb{N}$, larval helminths maturing in birds). (B) Relative proportions (percent of adult worms recovered) of lizard/reptile ( $\square$ ), amphibian $(\square)$ and bird $(\mathbb{\mathbb { N }})$ parasites.

Mean infracommunity similarity ranged from 0.067 to 0.855 across all samples and the higher similarity levels were consistently associated only with the presence of core species in the locality (partial $r=$ $0 \cdot 369, n=32, P<0 \cdot 05)$.

\section{Regional variations in community structure and composition}

Although there was some variation in the sample sizes (range 8-50), this was not significant in respect of the 3 regions under study. Therefore, initially, data from all samples were utilized in the comparisons of 
Table 2. The prevalence ( $\%$ infection) of helminth parasites in Lacerta agilis from 18 samples collected in the meadow steppeland zone in the Ukraine (Region 1)

\begin{tabular}{|c|c|c|c|c|c|c|c|c|c|c|c|c|c|c|c|c|c|c|}
\hline $\begin{array}{l}\text { Locality/sample no. } \\
\text { Sample size } \\
\text { Species }\end{array}$ & $\begin{array}{l}\text { N1 } \\
n=9\end{array}$ & $\begin{array}{l}\mathrm{N} 2 \\
n=13\end{array}$ & $\begin{array}{l}\mathrm{N} 3 \\
n=16\end{array}$ & $\begin{array}{l}\mathrm{N} 4 \\
n=50\end{array}$ & $\begin{array}{l}\text { N5 } \\
n=10\end{array}$ & $\begin{array}{l}\text { N6 } \\
n=9\end{array}$ & $\begin{array}{l}\mathrm{N} 7 \\
n=21\end{array}$ & $\begin{array}{l}\mathrm{N} 8 \\
n=13\end{array}$ & $\begin{array}{l}\text { N9 } \\
n=14\end{array}$ & $\begin{array}{l}\text { N10a } \\
n=38\end{array}$ & $\begin{array}{l}\text { N10b } \\
n=11\end{array}$ & $\begin{array}{l}\text { N10c } \\
n=10\end{array}$ & $\begin{array}{l}\text { N11 } \\
n=20\end{array}$ & $\begin{array}{l}\text { N12 } \\
n=8\end{array}$ & $\begin{array}{l}\text { N13a } \\
n=24\end{array}$ & $\begin{array}{l}\mathrm{N} 13 \mathrm{~b} \\
n=42\end{array}$ & $\begin{array}{l}\text { N14 } \\
n=15\end{array}$ & $\begin{array}{l}\mathrm{N} 15 \\
n=19\end{array}$ \\
\hline \multicolumn{19}{|l|}{ Trematoda } \\
\hline P. molini & - & - & - & - & - & - & - & - & - & - & - & - & $5 \cdot 0$ & - & - & - & - & - \\
\hline P. elegans & - & - & $12 \cdot 5$ & - & - & - & - & - & - & - & - & - & $25 \cdot 0$ & $37 \cdot 5$ & - & - & $6 \cdot 7$ & - \\
\hline P. medians & - & - & $6 \cdot 3$ & - & - & - & - & - & - & $10 \cdot 5$ & - & - & $40 \cdot 0$ & - & - & $2 \cdot 4$ & - & - \\
\hline P. confusus & - & - & - & - & - & - & - & - & - & - & - & - & - & - & - & - & - & $5 \cdot 3$ \\
\hline P. claviger & - & - & $6 \cdot 3$ & - & - & - & - & - & - & $2 \cdot 6$ & - & - & - & - & - & - & - & - \\
\hline Telorchis sp. & - & - & - & - & - & - & - & - & - & - & - & - & $5 \cdot 0$ & - & - & - & - & - \\
\hline \multicolumn{19}{|l|}{ Cestoda } \\
\hline O. tuberculata & - & - & $12 \cdot 5$ & - & - & - & - & - & - & $44 \cdot 7$ & $27 \cdot 3$ & $40 \cdot 0$ & $30 \cdot 0$ & - & - & - & - & $26 \cdot 3$ \\
\hline Nematotaenia sp. & - & - & - & $16 \cdot 0$ & - & - & - & - & - & - & - & - & - & - & - & - & - & - \\
\hline Mesocestoides spp. & - & - & - & - & - & $44 \cdot 4$ & - & - & - & - & - & - & - & - & - & - & - & - \\
\hline C. circi & - & - & - & - & - & - & - & - & - & - & - & $10 \cdot 0$ & - & - & - & - & - & - \\
\hline \multicolumn{19}{|l|}{ Nematoda } \\
\hline O. filiformis & - & - & - & - & - & - & - & - & - & - & - & - & - & - & - & - & $20 \cdot 0$ & - \\
\hline S. lacertae & - & - & - & - & - & - & - & - & $14 \cdot 3$ & $2 \cdot 6$ & - & - & - & - & - & - & - & $63 \cdot 2$ \\
\hline S. hoffmanni & - & - & $50 \cdot 0$ & - & $50 \cdot 0$ & - & - & - & - & - & - & - & - & - & - & - & - & - \\
\hline P. clausa & - & - & $6 \cdot 3$ & - & - & - & - & $15 \cdot 4$ & - & $34 \cdot 2$ & $18 \cdot 2$ & $70 \cdot 0$ & - & - & $16 \cdot 7$ & $11 \cdot 9$ & $13 \cdot 3$ & - \\
\hline S. contortus & - & - & - & - & - & - & - & - & - & $2 \cdot 6$ & - & - & $5 \cdot 0$ & - & - & - & - & - \\
\hline Habronema sp. & - & - & - & - & - & - & - & - & - & - & - & - & - & - & - & - & $6 \cdot 7$ & $10 \cdot 5$ \\
\hline P. sexalatus & $11 \cdot 1$ & - & - & - & - & - & - & - & - & - & - & - & - & - & - & - & - & - \\
\hline A. strongylina & - & - & - & - & - & - & - & $7 \cdot 7$ & - & - & - & - & - & - & - & - & - & - \\
\hline S. lupi & - & - & - & - & - & - & - & $7 \cdot 7$ & - & - & - & - & - & - & $4 \cdot 2$ & $2 \cdot 4$ & - & - \\
\hline C. obvelatus & - & - & - & - & - & - & $4 \cdot 8$ & - & - & - & - & - & - & - & - & - & - & - \\
\hline P. adunca & - & - & - & - & $20 \cdot 0$ & - & - & - & - & - & - & - & - & $12 \cdot 5$ & - & - & - & - \\
\hline P. cahirensis & $11 \cdot 1$ & - & - & - & - & - & - & $7 \cdot 7$ & - & - & $9 \cdot 0$ & - & - & - & $25 \cdot 0$ & $33 \cdot 3$ & - & - \\
\hline Acuariidae gen. sp. & - & $7 \cdot 7$ & $6 \cdot 3$ & $2 \cdot 0$ & - & $22 \cdot 2$ & - & - & $35 \cdot 7$ & - & - & $20 \cdot 0$ & - & $25 \cdot 0$ & - & $4 \cdot 8$ & - & - \\
\hline Acanthocephala & & & & & & & & & & & & & & 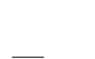 & & & & \\
\hline $\begin{array}{l}\text { S. picae } \\
\text { Centrorchynchus sp. }\end{array}$ & - & $7 \cdot 7$ & - & $22 \cdot 0$ & $20 \cdot 0$ & - & $\begin{array}{l}42 \cdot 9 \\
14 \cdot 3\end{array}$ & 100 & $57 \cdot 1$ & $\underline{10 \cdot 5}$ & $\begin{array}{r}9 \cdot 0 \\
-\end{array}$ & 一 & $\overline{10} \cdot 0$ & $\overline{62} \cdot 5$ & $33 \cdot 3$ & $47 \cdot 6$ & $53 \cdot 0$ & $26 \cdot 3$ \\
\hline M. moniliformis & - & - & - & - & - & - & - & - & - & - & - & - & - & - & $4 \cdot 2$ & - & - & - \\
\hline
\end{tabular}


Table 3. The prevalence (\% infection) of helminth parasites in Lacerta agilis from 14 samples collected in the grassland/forest transition zone in the Ukraine (Regions 2 and 3)

\begin{tabular}{|c|c|c|c|c|c|c|c|c|c|c|c|c|c|c|}
\hline \multirow{2}{*}{$\begin{array}{l}\text { Region } \\
\text { Locality/Sample no. } \\
\text { Sample size } \\
\text { Species }\end{array}$} & \multicolumn{9}{|c|}{ Middle Dnieper reaches (R2) } & \multicolumn{5}{|c|}{ Carpathian Mountains region (R3) } \\
\hline & $\begin{array}{l}\text { N1a } \\
n=21\end{array}$ & $\begin{array}{l}\text { N1b } \\
n=25\end{array}$ & $\begin{array}{l}\mathrm{N} 2 \\
n=14\end{array}$ & $\begin{array}{l}\text { N3 } \\
n=25\end{array}$ & $\begin{array}{l}\text { N4 } \\
n=17\end{array}$ & $\begin{array}{l}\text { N5 } \\
n=26\end{array}$ & $\begin{array}{l}\text { N6 } \\
n=15\end{array}$ & $\begin{array}{l}\text { N7 } \\
n=15\end{array}$ & $\begin{array}{l}\text { N8 } \\
n=14\end{array}$ & $\begin{array}{l}\text { N1 } \\
n=11\end{array}$ & $\begin{array}{l}\text { N2 } \\
n=19\end{array}$ & $\begin{array}{l}\text { N3 } \\
n=12\end{array}$ & $\begin{array}{l}\text { N4 } \\
n=44\end{array}$ & $\begin{array}{l}\text { N5 } \\
n=14\end{array}$ \\
\hline \multicolumn{15}{|l|}{ Trematoda } \\
\hline P. molini & - & - & - & $4 \cdot 0$ & $17 \cdot 7$ & - & - & - & - & $72 \cdot 7$ & $5 \cdot 3$ & $8 \cdot 3$ & $15 \cdot 9$ & - \\
\hline P. elegans & - & - & - & - & - & - & - & - & $7 \cdot 1$ & - & - & - & $2 \cdot 3$ & $7 \cdot 1$ \\
\hline P. maculosus & - & - & - & - & - & - & - & - & $7 \cdot 1$ & - & - & - & - & - \\
\hline P. medians & - & - & - & $4 \cdot 0$ & - & - & - & - & - & - & - & - & - & - \\
\hline Brachylaima sp. & - & - & - & - & $5 \cdot 9$ & - & - & - & - & $9 \cdot 1$ & - & - & - & - \\
\hline Strigea sp. & - & - & - & - & $5 \cdot 9$ & - & - & - & - & - & - & - & $2 \cdot 3$ & - \\
\hline A. alata & - & - & - & - & $5 \cdot 9$ & - & - & - & - & - & - & - & - & - \\
\hline \multicolumn{15}{|l|}{ Cestoda } \\
\hline Nematotaenia sp. & - & - & - & - & - & $3 \cdot 8$ & - & - & - & - & - & - & - & - \\
\hline Mesocestoides spp. & - & - & - & - & - & - & - & - & - & - & - & - & - & $7 \cdot 1$ \\
\hline $\begin{array}{l}\text { Nematoda } \\
\text { O. filiformis }\end{array}$ & - & $8 \cdot 0$ & $21 \cdot 4$ & $52 \cdot 0$ & $70 \cdot 6$ & $23 \cdot 1$ & - & $13 \cdot 3$ & $7 \cdot 1$ & $81 \cdot 8$ & 78.9 & $50 \cdot 0$ & $81 \cdot 8$ & 100 \\
\hline S. lacertae & - & - & $7 \cdot 1$ & - & - & - & - & - & - & - & - & - & - & - \\
\hline P. clausa & - & $4 \cdot 0$ & - & - & - & $3 \cdot 8$ & - & - & - & - & - & - & - & - \\
\hline P. sexalatus & $4 \cdot 8$ & $4 \cdot 0$ & - & - & - & - & - & - & - & - & - & - & - & $28 \cdot 6$ \\
\hline A. strongylina & $9 \cdot 5$ & - & - & - & $11 \cdot 8$ & - & $13 \cdot 3$ & - & - & - & $5 \cdot 3$ & $8 \cdot 3$ & $2 \cdot 3$ & - \\
\hline S. lupi & - & $4 \cdot 0$ & - & - & - & $3 \cdot 8$ & - & - & $21 \cdot 4$ & - & - & - & - & - \\
\hline Acuariidae gen. sp. & - & - & - & - & $11 \cdot 8$ & $3 \cdot 8$ & - & - & - & - & - & $8 \cdot 3$ & $2 \cdot 3$ & $7 \cdot 1$ \\
\hline $\begin{array}{l}\text { Acanthocephala } \\
\text { Centrorchynchus sp. }\end{array}$ & - & - & - & - & - & - & - & - & - & - & $5 \cdot 3$ & - & - & - \\
\hline
\end{tabular}


Table 4. Characteristics of the helminth communities in Lacerta agilis from the Ukraine

\begin{tabular}{|c|c|c|c|c|c|c|c|c|c|}
\hline $\begin{array}{l}\text { Parameters } \\
\text { Samples }\end{array}$ & $\mathrm{PUN} \dagger$ & TNS & $\mathrm{NCS}$ & NCOS & $\begin{array}{l}\text { Mean no. of } \\
\text { species (s.D.) }\end{array}$ & $\begin{array}{l}\text { Mean no. of } \\
\text { worms (s.D.) }\end{array}$ & Mean HB & Mean BP & $\begin{array}{l}\text { Mean } \\
\text { percentage } \\
\text { similarity }\end{array}$ \\
\hline \multicolumn{10}{|l|}{ Region 1} \\
\hline N1 & 0.78 & 2 & 0 & 0 & $0 \cdot 22(0 \cdot 44)$ & $1 \cdot 00(2 \cdot 1)$ & 0 & $1 \cdot 0$ & —* \\
\hline $\mathrm{N} 2$ & $0 \cdot 85$ & 2 & 0 & 0 & $0 \cdot 15(0 \cdot 38)$ & $0 \cdot 69(2 \cdot 2)$ & 0 & $1 \cdot 0$ & —* \\
\hline N3 & $0 \cdot 19$ & 7 & 3 & 1 & $1.00(0 \cdot 63)$ & $5 \cdot 81(6 \cdot 4)$ & $0 \cdot 073$ & $0 \cdot 94$ & $0 \cdot 395$ \\
\hline N4 & $0 \cdot 68$ & 3 & 2 & 0 & $0 \cdot 40(0 \cdot 64)$ & $0 \cdot 98(1 \cdot 8)$ & $0 \cdot 104$ & $0 \cdot 92$ & $0 \cdot 459$ \\
\hline N5 & $0 \cdot 30$ & 3 & 3 & 1 & $0.90(0.74)$ & $1 \cdot 30(1 \cdot 5)$ & $0 \cdot 128$ & $0 \cdot 86$ & $0 \cdot 524$ \\
\hline N6 & $0 \cdot 33$ & 2 & 2 & 0 & $0.67(0.50)$ & $645(794)$ & 0 & $1 \cdot 0$ & —* \\
\hline N7 & $0 \cdot 38$ & 3 & 2 & 0 & $0.62(0 \cdot 50)$ & $1 \cdot 19(1 \cdot 5)$ & 0 & $1 \cdot 0$ & $0 \cdot 500$ \\
\hline N8 & 0 & 5 & 1 & 1 & $1.38(0.87)$ & $14.8(13.9)$ & $0 \cdot 114$ & 0.94 & $0 \cdot 841$ \\
\hline N9 & $0 \cdot 21$ & 3 & 2 & 1 & $1.07(0.73)$ & $8 \cdot 86(13 \cdot 2)$ & $0 \cdot 127$ & $0 \cdot 86$ & $0 \cdot 427$ \\
\hline N10a & $0 \cdot 26$ & 7 & 4 & 0 & $1 \cdot 10(0 \cdot 89)$ & $4 \cdot 82(6 \cdot 4)$ & $0 \cdot 151$ & $0 \cdot 90$ & $0 \cdot 315$ \\
\hline $\mathrm{N} 10 \mathrm{~b}$ & $0 \cdot 45$ & 4 & 1 & 0 & $0.64(0.67)$ & $6 \cdot 73(12 \cdot 8)$ & $0 \cdot 067$ & 0.97 & $0 \cdot 144$ \\
\hline N10c & $0 \cdot 10$ & 4 & 3 & 1 & $1 \cdot 40(0 \cdot 70)$ & $4 \cdot 00(4 \cdot 3)$ & $0 \cdot 190$ & $0 \cdot 80$ & $0 \cdot 346$ \\
\hline N11 & $0 \cdot 25$ & 7 & 4 & 0 & $1 \cdot 20(0.95)$ & $5 \cdot 15(6 \cdot 7)$ & $0 \cdot 178$ & $0 \cdot 89$ & $0 \cdot 209$ \\
\hline N12 & 0 & 4 & 3 & 1 & $1.37(0.74)$ & $2.75(1.5)$ & $0 \cdot 121$ & $0 \cdot 88$ & $0 \cdot 268$ \\
\hline N13a & $0 \cdot 49$ & 5 & 3 & 0 & $0.83(0.92)$ & $20 \cdot 2(81 \cdot 4)$ & $0 \cdot 165$ & $0 \cdot 86$ & $0 \cdot 313$ \\
\hline N13b & $0 \cdot 31$ & 6 & 3 & 0 & $1.02(0.90)$ & $28 \cdot 7(113)$ & $0 \cdot 138$ & $0 \cdot 91$ & $0 \cdot 416$ \\
\hline N14 & $0 \cdot 40$ & 5 & 3 & 1 & $1 \cdot 00(1 \cdot 31)$ & $2 \cdot 80(4 \cdot 6)$ & $0 \cdot 212$ & $0 \cdot 88$ & $0 \cdot 450$ \\
\hline N15 & $0 \cdot 21$ & 5 & 4 & 1 & $1.32(0.95)$ & $8 \cdot 32(9 \cdot 1)$ & $0 \cdot 207$ & $0 \cdot 90$ & $0 \cdot 501$ \\
\hline \multicolumn{10}{|l|}{ Region 2} \\
\hline N1a & $0 \cdot 86$ & 2 & 0 & 0 & $0 \cdot 14(0 \cdot 36)$ & $0 \cdot 14(0 \cdot 4)$ & 0 & $1 \cdot 0$ & $0 \cdot 333$ \\
\hline N1b & $0 \cdot 88$ & 4 & 0 & 0 & $0 \cdot 20(0 \cdot 65)$ & $0 \cdot 28(1 \cdot 0)$ & $0 \cdot 200$ & $0 \cdot 87$ & $0 \cdot 067$ \\
\hline $\mathrm{N} 2$ & $0 \cdot 71$ & 2 & 1 & 0 & $0 \cdot 29(0 \cdot 47)$ & $1 \cdot 64(4 \cdot 5)$ & 0 & $1 \cdot 0$ & $0 \cdot 500$ \\
\hline N3 & $0 \cdot 48$ & 3 & 1 & 1 & $0.60(0.65)$ & $2 \cdot 16(3 \cdot 4)$ & $0 \cdot 059$ & 0.97 & $0 \cdot 855$ \\
\hline N4 & $0 \cdot 24$ & 7 & 4 & 1 & $1 \cdot 29(1 \cdot 21)$ & $6 \cdot 17(13 \cdot 2)$ & $0 \cdot 226$ & $0 \cdot 82$ & $0 \cdot 609$ \\
\hline N5 & $0 \cdot 69$ & 5 & 1 & 0 & $0 \cdot 38(0 \cdot 64)$ & $1 \cdot 04(2 \cdot 1)$ & $0 \cdot 106$ & $0 \cdot 89$ & $0 \cdot 446$ \\
\hline N6 & $0 \cdot 87$ & 1 & 1 & 0 & $0 \cdot 13(0 \cdot 35)$ & $0 \cdot 20(0 \cdot 6)$ & 0 & $1 \cdot 0$ & —* \\
\hline N7 & $0 \cdot 87$ & 1 & 1 & 0 & $0 \cdot 13(0 \cdot 35)$ & $0 \cdot 33(0 \cdot 9)$ & 0 & $1 \cdot 0$ & —* \\
\hline N8 & $0 \cdot 79$ & 4 & 1 & 0 & $0 \cdot 36(0.74)$ & $1 \cdot 29(3 \cdot 4)$ & $0 \cdot 223$ & $0 \cdot 84$ & $0 \cdot 153$ \\
\hline \multicolumn{10}{|l|}{ Region 3} \\
\hline N1 & 0 & 3 & 2 & 2 & $1 \cdot 64(0 \cdot 50)$ & $13 \cdot 5(13 \cdot 6)$ & $0 \cdot 275$ & $0 \cdot 81$ & $0 \cdot 518$ \\
\hline N2 & $0 \cdot 16$ & 4 & 1 & 1 & $0.95(0.52)$ & $6.95(7 \cdot 4)$ & $0 \cdot 044$ & 0.97 & $0 \cdot 819$ \\
\hline N3 & $0 \cdot 50$ & 4 & 1 & 1 & $0.75(0.87)$ & $4 \cdot 58(5 \cdot 5)$ & $0 \cdot 200$ & $0 \cdot 87$ & $0 \cdot 580$ \\
\hline N4 & $0 \cdot 14$ & 6 & 2 & 1 & $1.07(0.59)$ & $8 \cdot 48(9 \cdot 3)$ & $0 \cdot 105$ & $0 \cdot 91$ & $0 \cdot 741$ \\
\hline N5 & 0 & 5 & 2 & 1 & $1.50(0.52)$ & $9 \cdot 93(5 \cdot 7)$ & $0 \cdot 204$ & $0 \cdot 86$ & $0 \cdot 733$ \\
\hline
\end{tabular}

* Sample size too small to allow a meaningful value.

† PUN, proportion of uninfected hosts; TNS, total no. of species; NCS, no. of component species; NCOS, no. of core species; HB, Brillouin's diversity index; BP, Berger-Parker dominance index. Means for HB, BP and percentage similarity calculated for infected hosts only. The mean for percentage similarity is derived from all possible comparisons among lizards from each site in turn.

the helminth community characteristics across the regions (see Table 4 ).

At the component level, significant differences were found in the proportion of uninfected individuals in the samples $(\mathrm{K}-\mathrm{W} H=13 \cdot 23, \quad P=$ 0.0013). Samples taken from R2 had the highest proportions of uninfected lizards (range 0.24-0.88, median 0.79) while those from R3 and R1 showed more even levels of infection (ranges $0-0.50$ and $0-0 \cdot 85$, medians $0 \cdot 16$ and $0 \cdot 31$, respectively). A substantial regional variation was detected in the proportion of larval helminths in the component communities (K-W $H=9 \cdot 177, P=0 \cdot 0102$ ) with samples taken from R1 exhibiting the highest relative abundance of larval worms (median 0.73 ; 6 samples containing larval helminths only). Whilst the 3 regions did not differ in respect of total species richness, there was a significant difference between regions when component species were analysed (K-W $H=7 \cdot 27, P=0 \cdot 026$ ), primarily because of the discrepancy in values from R1 and R2 (medians 3 and 1 , respectively, Table 4 ).

There were more core species per sample in R3 (Table 4, K-W $H=8.98, P=0.011$, median 1) and this, coupled with variation in the distribution of the component species, resulted in significant variation in the mean infracommunity richness and abundance measures between the 3 regions $(\mathrm{K}-\mathrm{W} H=11 \cdot 37$, $P=0.0034$ and $H=11.09, P=0.0039$, respectively). Infracommunities in samples from R3 and R1 showed generally higher mean number of species and worms per individual lizard (medians 1.07 and 
Table 5. Means ( \pm s.D. $)$ and ranges for Jaccard Index and percentage similarity between communities sampled in the 3 regions in the Ukraine

\begin{tabular}{|c|c|c|c|c|c|c|}
\hline \multirow[b]{3}{*}{ Region } & \multicolumn{4}{|c|}{ Component communities } & \multirow{2}{*}{\multicolumn{2}{|c|}{$\frac{\text { Infracommunities }}{\text { Percentage similarity } f}$}} \\
\hline & \multicolumn{2}{|c|}{ Jaccard Index* } & \multicolumn{2}{|c|}{ Percentage similarity $\uparrow$} & & \\
\hline & Mean \pm s.D. & Range & Mean \pm s.D. & Range & Mean \pm s.D. & Range \\
\hline $\mathrm{R} 1$ & $0 \cdot 149 \pm 0 \cdot 13$ & $0-0 \cdot 67$ & $0 \cdot 159 \pm 0 \cdot 24$ & $0-0 \cdot 88$ & $0 \cdot 407 \pm 0 \cdot 16$ & $0 \cdot 14-0 \cdot 84$ \\
\hline $\mathrm{R} 2$ & $0 \cdot 162 \pm 0 \cdot 15$ & $0-0 \cdot 50$ & $0 \cdot 214 \pm 0 \cdot 23$ & $0-0 \cdot 79$ & $0 \cdot 423 \pm 0 \cdot 27$ & $0 \cdot 07-0 \cdot 86$ \\
\hline R3 & $0 \cdot 370 \pm 0 \cdot 11$ & $0 \cdot 15-0 \cdot 47$ & $0 \cdot 686 \pm 0 \cdot 28$ & $0 \cdot 34-0.94$ & $0 \cdot 678 \pm 0 \cdot 12$ & $0 \cdot 52-0 \cdot 82$ \\
\hline
\end{tabular}

* Calculated from presence/absence data on all parasites from each region irrespective of site.

$\uparrow$ Calculated from quantitative data on all parasites from each region irrespective of site.

I Calculated as the mean of the values in Table 4.

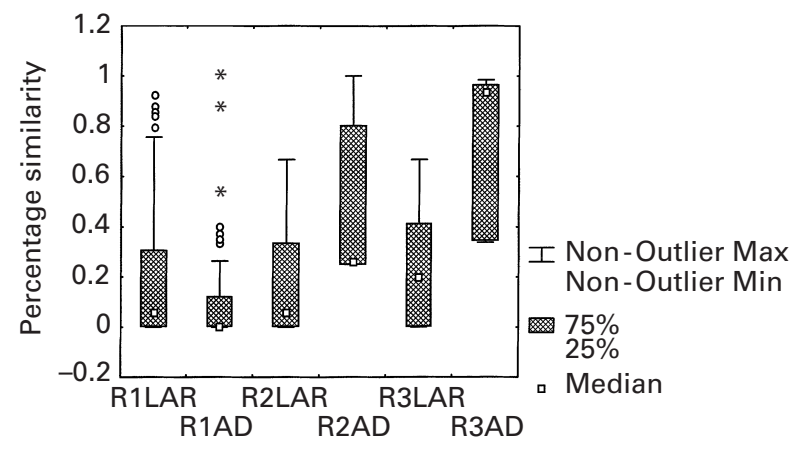

Fig. 4. Summary of percentage similarity indices, compared across component communities within each region sampled. Data are shown for larval (LAR) and adult (AD) helminths from communities sampled in Regions 1, 2 and 3 (R1, R2 and R3). The plot shows the median values $(\square)$, the $25-75 \%$ range (娄), the range for non-outlier values (error bars), and outliers (o) and extreme outliers $(*)$ plotted separately for each subset of data.

$1 \cdot 00$, and 8.48 and $4 \cdot 82$, respectively) as opposed to those sampled from R2 (medians 0.29 and 1.04, respectively).

Mean diversity and dominance values based on infracommunity data exhibited wide variation across the samples and showed no significant association with the region of host capture while the distributions of mean similarity differed between the 3 regions $(\mathrm{K}-\mathrm{W} H=6.99, P=0.030)$ with infracommunities sampled within R3 showing substantially higher similarity levels (median 0.733, Table 5).

However, after application of multiple regression to control for sample size, only 3 regional correlates (of the 7 identified above) remained significant $\left(R^{2}=\right.$ $\left.0.566, F_{3,27}=11.71, P=0.00004\right)$. These were 2 measures of component community structure, the proportions of larval worms $(\beta=-0.511, P=$ $0 \cdot 0013)$ and the number of component species $(\beta=-0.449, P=0.002)$, and the mean infracommunity similarity $(\beta=0.333, P=0 \cdot 029)$.

Significant regional variation in composition was also detected at the component community level.
The distributions of component community similarities differed between the 3 regions $(\mathrm{K}-\mathrm{W} H=13 \cdot 66$, $P=0.0011$ and $H=21.96, P=0.0001$ for Jaccard and percentage similarity index, respectively). In agreement with our findings at the infracommunity level, component communities sampled within R3 (Table 5) showed substantially higher similarity levels (median 0.869) while those from R1 were most dissimilar (median 0.05).

Similarity values in respect of larval helminths were consistently lower than those of adult helminths in the grassland/forest transition zone (mean 0.058 vs $0.261, P=0.0003$ and 0.202 vs 0.935 , $P=0.019$ for R2 and R3, respectively) as opposed to those from the meadow steppeland zone (R1) which, in spite of the wide range of variation and overlap, also differed significantly (mean 0.069 vs $0, P=$ $0 \cdot 0019$, Fig. 4). However, only the similarity values for adult helminths showed significant differences between regions $(\mathrm{K}-\mathrm{W} H=44 \cdot 09, P<0 \cdot 0001)$.

\section{DISCUSSION}

With a total of 30 helminth species identified, the exhaustive field collections gathered and analysed in this study almost certainly included the majority of the helminth fauna of sand lizards in the region. With few exceptions the helminth fauna of $L$. agilis in the Ukraine and Bulgaria reported in this study is consistent with the earlier report of Sharpilo (1976) who described 33 species from sand lizards collected in the former Soviet Union (including part of the present material). The exceptions comprised $O$. filiformis and Nematotaenia sp. which were previously identified as O. goezei Skrjabin \& Schulz, 1952 and $N$. tarentolae Lopez-Neyra, 1944, respectively, as well as the precise identification of the larval nematodes previously assigned to the composite group Agamospirura (spirurid nematodes with unclear taxonomic position, see Sharpilo, 1976). These were $P$. adunca (syn. Agamospirura biruchi Sharpilo, 1976) and C. obvelatus (syn. A. longioesophaga Sharpilo, 1963).

Our results therefore confirm that the sand lizard 
supports the richest helminth fauna among the Euroasian lacertids (see Sharpilo, 1976). However, significant spatial variation was encountered at different levels in this study. Thus the maximum total species richness was not achieved in any of the 3 large phytogeographical zones, the mountain zone in Bulgaria (only 2 species recovered), the meadow steppeland zone (26 species) and the grassland/forest transition zone (17 species) in the Ukraine. Differences were clearly apparent when the helminth fauna of $L$. agilis was compared qualitatively at a regional level, the 4 regions exhibiting similarity grouping corresponding to their location within the 3 phytogeographical zones. The Bulgarian data were the most distinct because of their extreme species poorness and were excluded from further analyses.

A characteristic feature of helminth component communities was the large proportion of heteroxeneous helminth species for which $L$. agilis (as in many reptile species, see Sharpilo, 1983) serves as a paratenic host, and to a much lesser degree, as intermediate host. The substantial differences between the 3 regions emphasize the importance of the local compound community (supra community) structure since both the diversity and abundance of larval helminths depends on overlap in the host range with other vertebrates (mammals and birds) that support the species in the region. Thus the higher abundance of avian parasites ( $P$. elegans, larval Acuariidae and cystacanths of $S$. picae and Centrorhynchus sp.) in sand lizards from the meadow steppeland region (R1) is most likely a mass-effect (Shmida \& Wilson, 1985) linked to the greater availability of host birds in R1 compared with R2 and R3. Almost all lizards in R1 were collected in an area encompassing a number of natural reserves (see Appendix) which support both diverse and abundant avian communities.

Surprisingly, species with presumably higher colonization abilities (or greater dispersal potential) in respect of host vagility (i.e. larval helminths with avian or mammalian final hosts) contributed very little to the similarity in composition between component communities of lizard helminths even within the same region. Thus, although the similarity levels due to larval helminths between samples from $\mathrm{R} 1$, which was characterized by the highest richness and local abundance of larval forms, were significantly higher than those due to adult helminths, they both exhibited consistently low values. This observation contrasts with the predictions and patterns recognized in many helminth communities of freshwater fish (see Esch et al. 1998; Poulin, 1997). The only plausible explanation for the low levels of predictability of component communities in L. agilis (and probably in all reptile species) with respect to larval helminths is evolutionary i.e. the non-obligatory and hence highly stochastic nature of paratenic parasitism.
Another interesting feature of the helminth component communities of sand lizards was the absence of strict host specificity (see also Aho, 1990) since all the helminths recovered in this study were species which sand lizards share either with other reptile hosts or with amphibians and birds. Moreover, the present results confirm the conclusion from our recent study on another European lacertid host, Lacerta viridis, that communities from marginal habitats are dominated by host generalist species while those from typical habitats are dominated by lizard specialist (Biserkov \& Kostadinova, 1998). Although the sand lizard has a wide European distribution which extends to central Asia and is tolerant of a wide range of habitat conditions, it is considered to have originated in the steppes (Yablokov et al. 1981; Frazer, 1989) and the typical meadow steppelands here (R1) are representative of its preferred habitats. Consistent with this view, we found that L. agilis was parasitized by a much higher proportion of lizard specialists in the localities of the meadow steppeland zone (R1). Although the identity of the lizard specialists which attained core status in the communities from typical habitats differed between the two hosts ( $L$. agilis and L. viridis), the amphibian/reptile generalist $O$. filiformis was the most frequent and abundant species in the marginal habitats in both studies.

While there were very clear differences in the component community structures of lizards from different phytogeographical zones, analysis of similarity patterns at both component and infracommunity levels suggests that local effects are equally important e.g. host population/habitat. The significant differences in the between-component community and between-infracommunity similarities observed in the 3 Ukranian regions suggest 2 types of communities that vary in their predictability with respect to species composition in relation to the regional helminth fauna. Communities sampled from the meadow steppeland region (R1) were less predictable as opposed to those sampled from the Carpathian Mountains region (R3) which showed high and comparable quantitative similarity at both levels. The range of the geographical habitats sampled in R1 and the highest regional total species richness observed (26 species) suggest that low similarity may have arisen through the availability of a larger range of final hosts serving as sources of infection for sand lizards. In contrast, the sampling area in the Carpathian Mountains region was relatively small and homogeneous with respect to habitat characteristics. The regional total species diversity was low (10 species), and the high similarity between the local lizard helminth component communities most likely arose through the availability of the amphibian/reptile generalist $O$. filiformis, a species with a direct life-cycle.

A different picture was revealed by the helminth 
component communities in sand lizards sampled from the Middle Dnieper reaches (R2) (low regional diversity, 13 species, and area comparable to R3). Although the component communities were close in composition and structure to those from R3, their position in terms of the similarity scale was much closer to those sampled in R1. In this case, largely unexplained stochastic effects, probably related to the human pressure and loss of habitat quality, may have affected helminth communities, giving rise to the highest proportion of uninfected individuals and the lowest mean infracommunity richness and abundance.

Overall, the wide range of variation observed in both the composition and structure of the helminth component communities in L. agilis in the Ukraine, suggests that spatial effects have an important influence on patterns observed in the host-parasite system and that they are linked to typical versus marginal habitats of the host. Our results indicate that (i) clear regional differences in composition of component communities exist and that they affect the degree of predictability of infracommunities, and (ii) the structure of infracommunities does not show marked regional variation i.e. these were essentially depauperate infracommunities with high homogeneity of structural parameters irrespective of species composition or region of origin. The presence and the distribution of the core species indicated that we had sampled mostly isolated lizard populations in which a restricted number of species have become locally abundant and this facilitated an increased predictability at the infracommunity level. Finally, in line with our expectations, parasite communities of lizards from typical habitats were dominated by lizard specialists, whilst those from hosts sampled in marginal habitats were characterized by host generalist species. However, in contrast to our expectations for predictability, helminth communities of lizards living in marginal habitats exhibited a higher homogeneity of composition than those sampled from typical habitats. These results strongly suggest that host specificity is not an important factor is setting the simple structure of this host-parasite system.

This study is a result of a joint project between the Bulgarian Academy of Sciences and the National Academy of Sciences of the Ukraine.

\section{REFERENCES}

AHO, J. M. (1990). Helminth communities of amphibians and reptiles: comparative approaches to understanding patterns and processes. In Parasite Communities:

Patterns and Processes (ed. Esch, G. W. Bush, A. O. \& Aho, J. M.), pp. 157-190. Chapman and Hall, London and New York.

BAEv, P. \& PENEV, L. (1995). BIODIV. Program for Calculating Biological Diversity Parameters, Similarity,
Niche Overlap, and Cluster Analysis. Version 5.1 Exeter Software, New York.

BISERKov, v. \& Kostadinova, A. (1998). Intestinal helminth communities in the green lizard, Lacerta viridis, from Bulgaria. Fournal of Helminthology 72, $267-271$.

Bush, A. O. (1990). Helminth communities in avian hosts: determinants of pattern. In Parasite Communities: Patterns and Processes (ed. Bush, G. W. \& Aho, J. M.), pp. 197-232. Chapman and Hall, London.

BUSH, A. O., AHO, J. M. \& KENNEDY, C. R. (1990). Ecological versus phylogenetic determinants of helminth parasite community richness. Evolutionary Ecology 4, 1-20.

BUSh, A. O., LAFFERTY, K. D., LOTZ, J. M. \& Shostak, A. W. (1997). Parasitology meets ecology on its own terms: Margolis et al. revisited. Fournal of Parasitology 83, 575-583.

ESCH, G. W., Bush, A. O. \& AHo, J. M. (1990). Parasite Communities: Patterns and Processes. Chapman and Hall, London and New York.

FRAZER, D. (1989). Reptiles and Amphibians in Britain. Bloomsbury Books, London.

ESCH, G. W., KENNEDY, C. R., BUSH, A. O. \& AHO, J. M. (1988). Patterns in helminth communities in freshwater fish in Great Britain: alternative strategies for colonization. Parasitology 96, 519-532.

JANOvy, J. JR., ClOpton, R. E. \& PERCIVAL, T. J. (1992). The roles of ecological and evolutionary influences in providing structure to parasite species assemblages. Fournal of Parasitology 78, 630-640.

KREBS, C. J. (1989). Ecological Methodology. Harper Collins Publishers, New York.

LEwin, J. (1992). Parasites of the sand lizard (Lacerta agilis L.) in Poland. Acta Parasitologica 37, 19-24.

MagurRan, A. E. (1988). Ecological Diversity and its Measurement. Croom Helm Ltd, London.

POULIN, R. (1997). Species richness of parasite assemblages: evolution and patterns. Annual Review of Ecology and Systematics 28, 341-358.

POUlin, R. (1998 a). Evolutionary Ecology of Parasites. From Individuals to Communities. Chapman and Hall, London, $212 \mathrm{pp}$.

POUlin, R. $(1998 b)$. Comparison of three estimators of species richness in parasite component communities. Fournal of Parasitology 84, 485-490.

Sharpilo, v. P. (1976). [Parasitic Worms of the Reptilian Fauna of the USSR: Systematics, Chorology, Biology]. Naukova Dumka, Kiev. (In Russian).

SHARPILO, v. P. (1983). [Reptiles of the fauna of the USSR, intermediate and reservoir hosts of helminths]. Parazitologiya 17, 177-184 (In Russian).

SHMIDA, A. \& WILSON, M. v. (1985). Biological determinants of species diversity. Fournal of Biogeography 12, 1-20.

SOKAL, R. R. \& ROHLF, F. J. (1981). Biometry. The Principles and Practice of Statistics in Biological Research, 2nd Edn. W. H. Freeman and Co., New York.

YABlokov, A. V., BARANOV, A. S. \& ROZANOV, A. s. (1981). [Species microphylogenesis reconstruction: a study on the Sand Lizard, L. agilis]. Vestnik Zoologii, 3, 11-16. (In Russian). 
APPENDIX

Locations of the study sites and dates on which sand lizards were sampled.

Localities in the Ukraine:

Region 1, Meadow steppeland region (Black and Azov Sea coasts and short grass steppes):

$\mathrm{N} 1$, village Reni, Odessa district, $30^{\circ} 00^{\prime} \mathrm{N}, 28^{\circ} 25^{\prime} \mathrm{E}, 07.1951$;

N2, Lagernaya kosa, Nikolaevsk district, $46^{\circ} 40^{\prime} \mathrm{N}, 31^{\circ} 35^{\prime} \mathrm{E}, 07.1977$;

N3, Island Tendra, Black Sea Reserve, Kherson district, $46^{\circ} 20^{\prime} \mathrm{N}, 31^{\circ} 33^{\prime} \mathrm{E}, 08.1964$;

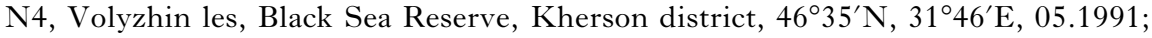

N5, Solenoozernyi uchastok, Black Sea Reserve, Kherson district, $46^{\circ} 30^{\prime} \mathrm{N}, 31^{\circ} 55^{\prime} \mathrm{E}, 06.1991$;

N6, Yagorlytskii kut, Black Sea Reserve, Kherson district, $46^{\circ} 20^{\prime} \mathrm{N}, 31^{\circ} 45^{\prime} \mathrm{E}, 07.89$;

N7, Ivano-Rybal'chanskii uchastok, Black Sea Reserve, Kherson district, $46^{\circ} 29^{\prime} \mathrm{N}, 32^{\circ} 03^{\prime} \mathrm{E}, 05.1990$;

$\mathrm{N} 8$, village Chernyanka, Kherson district, $46^{\circ} 25^{\prime} \mathrm{N}, 32^{\circ} 23^{\prime} \mathrm{E}, 05.1961$;

N9, Askania Nova Reserve, Kherson district, 46 $33^{\prime} \mathrm{N}, 34^{\circ} 10^{\prime} \mathrm{E}, 04.1972$;

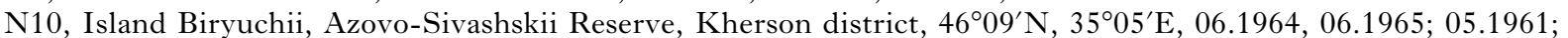

N11, Obitochnaya kosa, Kherson district, $46^{\circ} 33^{\prime} \mathrm{N}, 36^{\circ} 11^{\prime} \mathrm{E}, 07.1964$;

N12, Berdyanskaya kosa, Kherson district, $46^{\circ} 38^{\prime} \mathrm{N}, 36^{\circ} 48^{\prime} \mathrm{E}, 07.1977$;

N13, Kamennye Mogily Reserve, Donetsk district, $47^{\circ} 20^{\prime} \mathrm{N}, 37^{\circ} 25^{\prime} \mathrm{E}, 07.1964 ; 05.1965$;

N14, Khomutovskaya step' Reserve, Donetsk district, $47^{\circ} 20^{\prime} \mathrm{N}, 38^{\circ} 10^{\prime} \mathrm{E}, 06.1963$;

N15, Streletskaya step' Reserve, Lugansk district, $49^{\circ} 50^{\prime} \mathrm{N}, 44^{\circ} 05^{\prime} \mathrm{E}, 06.1963$.

Region 2, Middle Dnieper reaches along Kiev's and Kanev's dams:

$\mathrm{N} 1$, Brannoe pole, Kiev district, $49^{\circ} 29^{\prime} \mathrm{N}, 30^{\circ} 45^{\prime} \mathrm{E}, 06.1956,05.1955$;

N2, village Dmitrenki, Kiev district, $49^{\circ} 30^{\prime} \mathrm{N}, 30^{\circ} 45^{\prime} \mathrm{E}, 04.1983$;

N3, Kanev Reserve, Cherkask district, $49^{\circ} 42^{\prime} \mathrm{N}, 31^{\circ} 23^{\prime} \mathrm{E}, 06.1955$;

N4, Kanev-Zarech'E, Cherkask district, $49^{\circ} 50^{\prime} \mathrm{N}, 31^{\circ} 27^{\prime} \mathrm{E}, 06.1955$;

N5, Pushchevoditsa, Kiev district, $50^{\circ} 31^{\prime} \mathrm{N}, 30^{\circ} 20^{\prime} \mathrm{E}, 06.1951$;

N6, village Semiyapolki, Chernigovsk district, $51^{\circ} 20^{\prime} \mathrm{N}, 31^{\circ} 27^{\prime} \mathrm{E}, 05.1951$;

N7, village Vil'cha, Kiev district, $51^{\circ} 19^{\prime} \mathrm{N}, 29^{\circ} 25^{\prime} \mathrm{E}, 04.1960$;

N8, village Novoshepel'che, Kiev district, $51^{\circ} 21^{\prime} \mathrm{N}, 30^{\circ} 06^{\prime} \mathrm{E}, 08.1951$.

\section{Region 3, Carpathian Mountains region:}

$\mathrm{N} 1$, village Iza, Zakarpatsk district, $48^{\circ} 14^{\prime} \mathrm{N}, 23^{\circ} 17^{\prime} \mathrm{E}, 07.1961$;

$\mathrm{N} 2$, village Vorokhta, Ivano-Frankovsk district, $48^{\circ} 16^{\prime} \mathrm{N}, 24^{\circ} 32^{\prime} \mathrm{E}, 06.1957$;

$\mathrm{N} 3$, village Delyatin, Ivano-Frankovsk district, $48^{\circ} 32^{\prime} \mathrm{N}, 24^{\circ} 38^{\prime} \mathrm{E}, 06.1957$;

N4, village Nezhukhov, L'vovsk district, $49^{\circ} 15^{\prime} \mathrm{N}, 2^{\circ} 45^{\prime} \mathrm{E}, 06.1957$;

$\mathrm{N} 5$, village Ivan-Franko, L'vovsk district, $49^{\circ} 55^{\prime} \mathrm{N}, 23^{\circ} 47^{\prime} \mathrm{E}, 08.1961$.

Localities from Bulgaria

Yavorov hut, Pirin Mountains, $42^{\circ} 05^{\prime} \mathrm{N}, 23^{\circ} 45^{\prime} \mathrm{E}, 08.1984$, 08.1985;

village Razlog, Pirin Mountains, $42^{\circ} 00^{\prime} \mathrm{N}, 23^{\circ} 40^{\prime} \mathrm{E}, 08.1985$. 